\title{
Impact of Bandwidth on Small-Scale Fade Depth
}

\author{
Wasim Q. Malik, Ben Allen, and David J. Edwards \\ Department of Engineering Science, University of Oxford \\ Parks Road, Oxford, OX1 3PJ, United Kingdom \\ Email: \{wasim.malik, ben.allen, david.edwards\}@eng.ox.ac.uk
}

\begin{abstract}
This paper investigates the impact of channel bandwidth on fading in wireless channels using indoor measurements. The variation of channel energy over a local region is examined for narrowband, wideband and ultrawideband (UWB) channels, and the corresponding fade depth is evaluated. The relation between bandwidth and fade depth is then captured with a simple dual-slope model. The effect of antenna polarization and line-ofsight blockage is also investigated. We observe that the fade depth initially falls rapidly with bandwidth, reaching $4 \mathrm{~dB}$ at $1 \mathrm{GHz}$, but further reduction in fading with bandwidth is much slower.
\end{abstract}

\section{INTRODUCTION}

Multipath propagation in wireless channels causes destructive signal interference and signal fading, which is a serious detriment to reliable communications [1]. Unresolved multipath components (MPCs) give rise to severe fading, leading to a high system outage probability. The link budget then requires a large fade margin to compensate for the fading and ensure the desired quality of service. Fading is closely related to the spatio-temporal variability of the channel. In slow-fading indoor channels, the rapid spatial variation of the received signal levels over a small-scale region is usually dominant [1]. Wideband and ultrawideband (UWB) systems can explot their inherent frequency diversity to mitigate fading with the use of appropriate signal processing techniques [2], [3].

In narrowband channels, fading generally leads to Rayleigh, Ricean or Weibull distributed channel amplitude statistics [1]. Some recent work has investigated the impact of bandwidth on channel fading using information theoretic and statistical approaches. The number of significant eigenvalues of the channel covariance matrix, obtained from the channel impulse response, was shown to scale linearly with bandwidth in [4], while [5] reported that a saturation point is reached at a certain bandwidth. These results indicate an increase in the stochastic degrees of freedom with bandwidth in frequency-selective channels, leading to reduced fading. Based on the Nakagami$m$ parameter estimation approach, the channel robustness was found to increase substantially with bandwidth in [6].

In this paper, the fade depth refers to the variation of the received symbol energy about its local mean due to smallscale fading. Owing to the central importance of this quantity in radio network planning, a number of fade depth prediction models have been presented. The empirically obtained OlsenSegal fade depth prediction model has been adopted by ITU$\mathrm{R}$ for terrestrial point-to-point LOS links [7]. The impact of bandwidth on fade depth for up to $10 \mathrm{MHz}$ wide codedivision multiple access (CDMA) channels was reported in
[8]. To our knowledge, however, no model is available that relates the small-scale fade depth to bandwidth over a large bandwidth range. This vacuum motivates the analysis in this paper, undertaken over the FCC-allocated UWB band [3], encompassing narrowband, wideband and UWB channels. Such characterization is especially important for practical wireless systems, as it would allow the system designer to determine the optimal operating bandwidth and required link budget with the desired level of performance-complexity tradeoff.

The contribution of this paper is a simple empirical model relating fade depth to channel bandwidth. The propagation scenarios consider both line-of-sight (LOS) and non-lineof-sight (NLOS) propagation. The effect of polarization is investigated by analyzing both vertical and horizontal link polarizations as defined by the antenna orientation.

\section{System Model And Channel Measurement}

For a linear multipath channel, the time-domain channel impulse response (CIR), $h(\tau)$, and the frequency-domain channel transfer function (CTF), $H(f)$, form a Fourier transform pair, where $\tau$ denotes the time-delay with respect to the first path. The baseband CTF can be expressed in the form

$$
H(f)=\mathcal{F}\{h(\tau)\}=\sum_{k=0}^{N_{f}-1} \alpha_{k} e^{j \phi_{k}} \delta(f-k \Delta f),
$$

where $\alpha_{k}$ and $\phi_{k}$ are the magnitude and phase responses of the channel at the $k^{\text {th }}$ frequency point, $N_{f}$ is the number of frequency points in the CTF, $\Delta f=W /\left(N_{f}-1\right)$ is the frequency resolution, $W$ is the channel bandwidth, and $\mathcal{F}\{\cdot\}$ denotes the discrete Fourier transform operation. From the Parseval relation, the wideband channel energy is

$$
E=\int_{0}^{\infty}|h(\tau)|^{2} \mathrm{~d} \tau=\int_{-\infty}^{\infty}|H(f)|^{2} \mathrm{~d} f .
$$

Channel measurements are conducted to obtain a large ensemble of CTFs in a set of indoor small-office locations, as shown in Fig. 1. Each location presents a dense scattering environment with various objects such as computers, cabinets, furniture, and other equipment. Within each room, measurements are performed at two different locations, so that a total of four sets of small-scale measurements are obtained. The corresponding locations of the transmitter-receiver pairs are represented in Fig. 1 by $T_{i}$ and $R_{i}, i=1, \ldots, 4$. For each measurement set, the location of the transmitting antenna is fixed, while the receiving antenna, controlled by an automated 

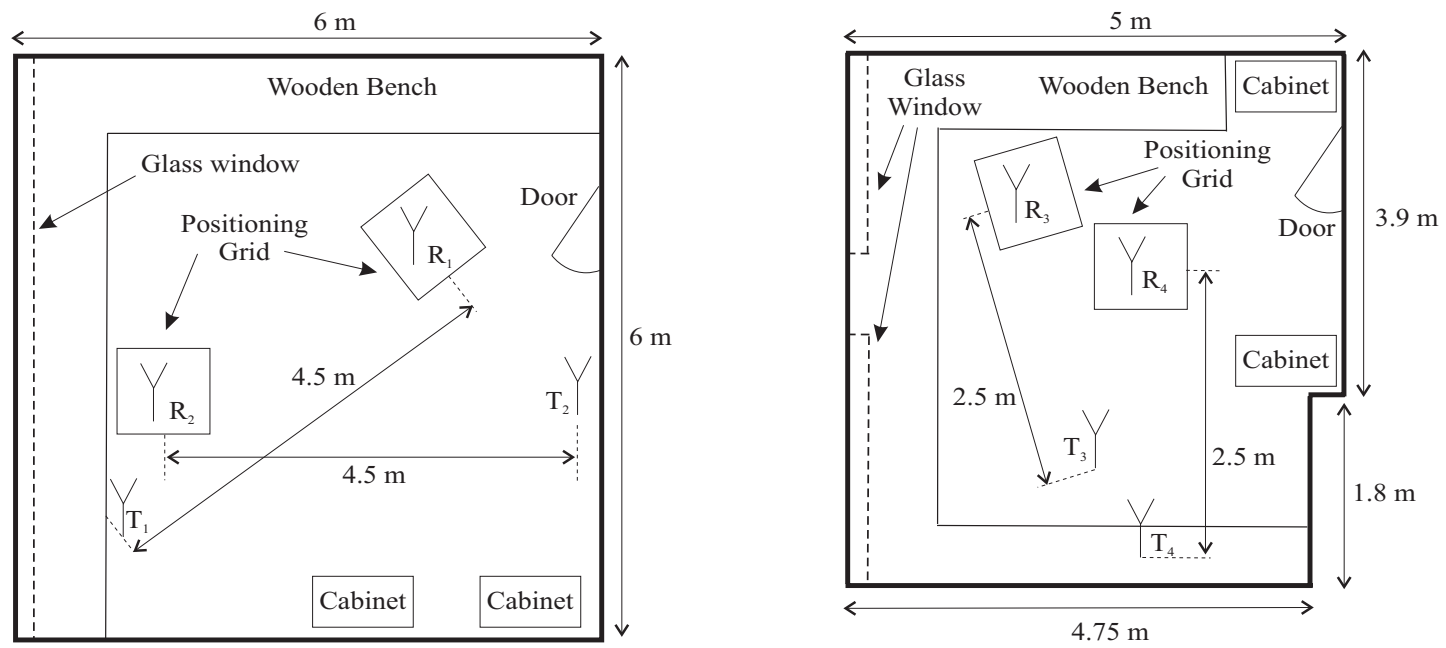

Fig. 1. The indoor propagation environment and measurement configuration. The measured links are defined by the $T_{i} R_{i}$ antenna pair, $i=\{1, \ldots, 4\}$.

positioning grid, is placed at $N_{i}=900$ separate locations within a $1 \mathrm{~m}^{2}$ area. The successive locations are chosen to be $0.03 \mathrm{~m}$ apart in each direction of the grid in order to obtain sufficiently spatially uncorrelated channel responses. In the rest of this paper, the CTF at the $j^{\text {th }}$ location within the $i^{\text {th }}$ grid dataset is denoted by $H(i, j, f)$. Accordingly, we have the CIR $h(i, j, \tau)$, the frequency-domain amplitude $\alpha_{k}(i, j)$, and the channel energy $E(i, j)$. The transmitting and receiving antennas are placed at the same height of $1.5 \mathrm{~m}$. These measurements are undertaken for both LOS and NLOS propagation scenarios. A large block of RF-absorbent material is placed between the transmitting and receiving antennas to create the NLOS scenario. The propagation environment is physically isolated for the duration of the measurement.

We also analyze the impact of signal polarization on fading in this paper. To measure the vertically polarized (VV) link, the transmitting and receiving antennas are placed vertically. For the measurement of the horizontally polarized (HH) link, the antennas are rotated by $90^{\circ}$ so that the antenna axis is perpendicular to the line joining the transmitter and receiver.

Using a vector network analyzer (VNA) arrangement [9], the complex transmission parameter, $S_{21}(f)$, is measured between $f_{l}=3.1 \mathrm{GHz}$ and $f_{h}=10.6 \mathrm{GHz}$ at $N_{f}=1601$ frequency points. The bandwidth is thus $W=f_{h}-f_{l}=7.5 \mathrm{GHz}$ and frequency resolution is $\Delta f=4.685 \mathrm{MHz}$. End-to-end system calibration is performed followed by the compensation of the measured channel responses. Omni-directional UWB antennas with the discone construction are used [10], [11].

The local mean pathloss is removed from each channel response within the dataset for each location. The pathloss normalization factor, $F_{i}$, for the $i^{\text {th }}$ dataset is calculated as $L_{i}=\sqrt{\left(1 / N_{i}\right) \sum_{j=1}^{N_{i}} E(i, j)}$. Each $H(i, j, f)$ is then divided by $L_{i}$. As a representative example, the CTF, $H(\mathbf{r}, f)$, measured at the center of the grid in the $T_{1} R_{1}$ dataset is shown in Fig. 2, where $\mathbf{r}=(i, j)$. The frequency selectivity of the

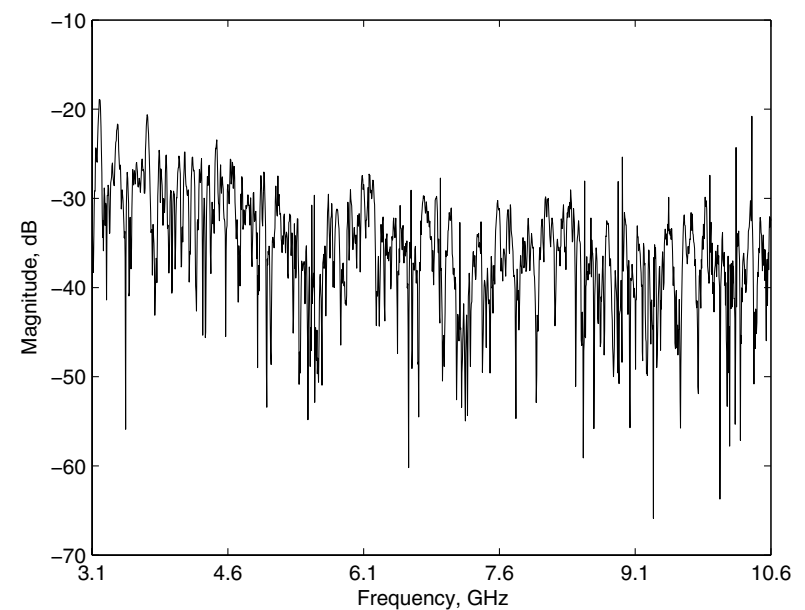

Fig. 2. A measured UWB channel transfer function over the FCC band, 3.1-10.6 GHz, as an example.

UWB channel is obvious, with observed coherence bandwidth on the order of $W_{c}=30 \mathrm{MHz}$.

\section{FADE Depth Evaluation}

In this section, we investigate the variation of channel energy and fade depth with bandwidth. The comparative analysis is undertaken for narrowband, wideband and UWB channels over a range of bandwidths. The frequency-sectioned CTF, $H_{b}(\mathbf{r}, f)$, with bandwidth $W_{b}$, is obtained from $H(\mathbf{r}, f)$ by considering the appropriate number of measured frequency points, $n_{f} \in\left\{1, \ldots, N_{f}\right\}$. Thus the channel bandwidth, $W_{b}=$ $n_{f} \Delta f$, varies in our case from an arbitrarily small value for a pure sinusoid to $7.5 \mathrm{GHz}$ for a full-band UWB signal. The center frequency is maintained at $f_{c}=\left(f_{l}+f_{h}\right) / 2=$ $6.85 \mathrm{GHz}$ for fair comparison, and the measured discrete center frequency is denoted by $n_{c}$. The bandwidth is altered 


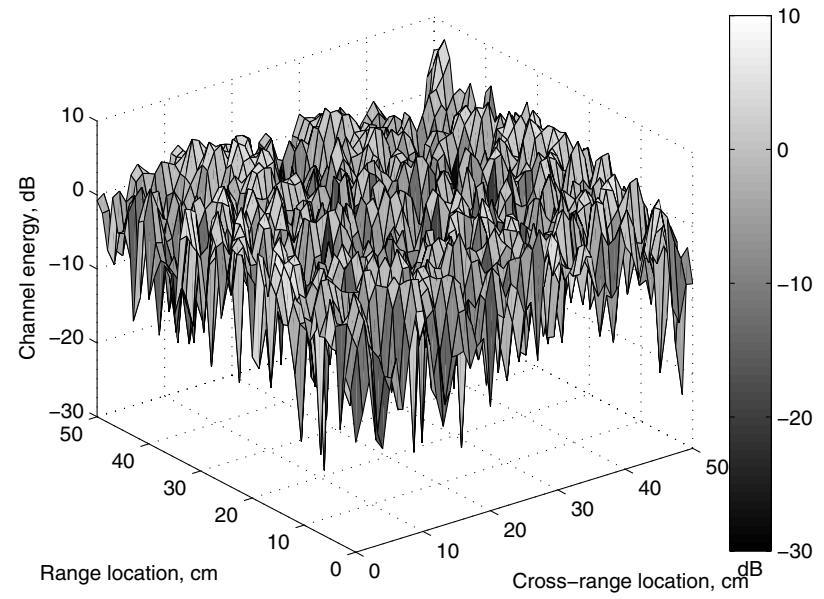

(a) Narrowband channel $(6.85 \mathrm{GHz})$

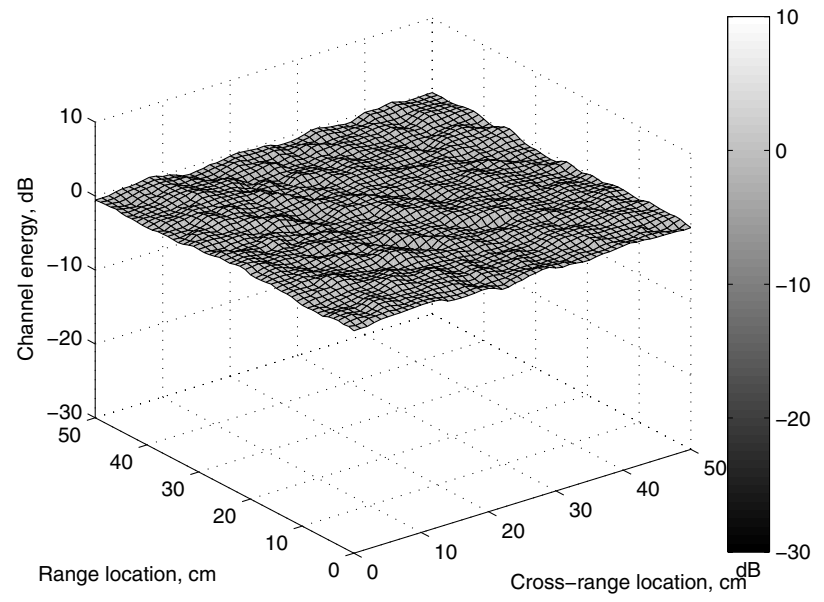

(b) Ultrawideband channel $(3.1-10.6 \mathrm{GHz})$

Fig. 3. Small-scale fading in the $T_{1} R_{1}$ LOS environment illustrated by the spatial variation of channel energy normalized to the local mean energy.

by including $n_{i}=\left(n_{f}-1\right) / 2$ measured frequency points symmetrically distributed on either side of $n_{c}$, assuming odd $n_{f}$ without loss of generality. Then the band-limited CTF is

$$
H_{b}(\mathbf{r}, f)= \begin{cases}H(\mathbf{r}, f), & \text { if }\left|f-f_{c}\right| \leq W_{b} / 2 \\ 0, & \text { otherwise. }\end{cases}
$$

As the measurement data is obtained in the frequency domain, the following analysis is also undertaken in the same domain. By the Parseval relation in (2), this frequency-domain energy integration is equivalent to multipath energy integration using a maximal-ratio combining (MRC) rake receiver or allrake. The energy of the band-limited channel, $H_{b}$, is thus

$$
E_{b}(\mathbf{r})=\int_{f_{l}}^{f_{h}}\left|H_{b}(\mathbf{r}, f)\right|^{2} \mathrm{~d} f .
$$

Note that, strictly speaking, $W_{b}=0$ when $n_{i}=0$. Since $\lim _{W_{b} \rightarrow 0} \log _{10} W_{b}=-\infty$, we substitute the effective bandwidth, $W_{b}=\Delta f$, in this case for mathematical convenience. This allows the inclusion of the single-frequency sample point, $n_{c}$, corresponding to the center frequency, $f_{c}$, in the logbandwidth plot and model. There is no loss of generality since $\Delta f<<W_{c}$, and therefore the fading at $n_{c}$ is flat over $\Delta f$.

For the indoor multipath channel, the fade depth is a measure of the variation in the channel energy due to small-scale fading. Indoor wideband channels are predominantly affected by spatial fading, while in outdoor environments, temporal variability also contributes to fading. Fig. 3 illustrates the small-scale spatial variation of the measured channel energy for indoor narrowband and UWB channels. It is apparent that the narrowband channel is far more susceptible to multipath interference and small-scale fading than the UWB channel.

The fade depth, $F$, is defined as the observed range of signal power variation. Statistically, the fade depth is related to the standard deviation, $\sigma$, of the channel energy expressed in $\mathrm{dB}$ scale. In order to cover a range of system performance levels,

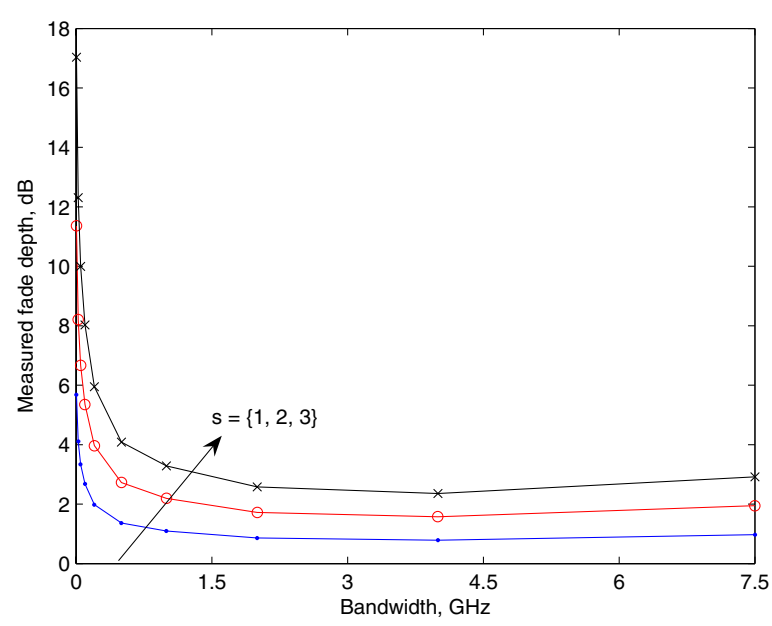

Fig. 4. The measured fade depth, $F_{s \sigma}$, as a function of channel bandwidth for various values of $s$.

$F$ is defined in this treatment as the $\sigma, 2 \sigma$, and $3 \sigma$ levels, and is denoted by $F_{s \sigma}$, where $s=\{1,2,3\}$. The fade depths are estimated in this manner for a variety of channel bandwidths. As shown in Fig. 4 for the NLOS VV channel, it is observed that $F_{s \sigma}=\{5.7,11.3,17.0\} \mathrm{dB}$ at $s=\{1,2,3\}$, respectively, at $W_{b}=\Delta f=4.685 \mathrm{MHz}$. On the other hand, we observe $F_{s \sigma}=\{1.0,1.9,2.9\} \mathrm{dB}$ at $s=\{1,2,3\}$, respectively, with $W_{b}=7.5 \mathrm{GHz}$. A comparison of the corresponding values shows that the transition from the narrowband to the UWB channel leads to an almost six-fold reduction in fade depth. Thus clearly $W_{b}$ has a very significant impact on $F_{s \sigma}$.

The $F_{s \sigma}$ curves appear to converge to their asymptotic values at approximately $1 \mathrm{GHz}$, which is in agreement with the results in [12]. A further increase in $W_{b}$ yields only a minor reduction in $F_{s \sigma}$, and no performance improvement with larger 


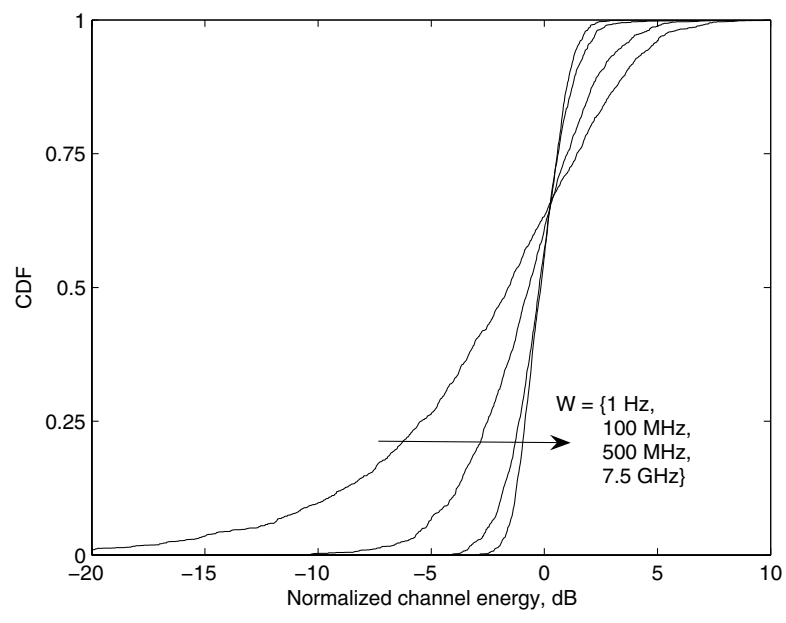

Fig. 5. CDFs of channel energy, normalized with respect to the small-scale local mean, for various values of the channel bandwidth, $W$.

$W_{b}$ can be expected once the fade margin reaches a constant value. The saturation of the multipath resolution capability of wideband systems has also been reported in [5], [13].

These observations indicate that the additional resilience to fading from bandwidths beyond approximately $1 \mathrm{GHz}$ is insignificant. However, a further increase in bandwidth beyond this critical point may still continue to improve the spatial resolution important for localization and imaging systems [3].

\section{FADE DEPTH MODEL}

A simple empirical model for the estimation of fade depth as a function of channel bandwidth is now proposed. Fig. 6 shows the measured fade depth, evaluated earlier in Sec. III, plotted against the bandwidth on a logarithmic scale. The observation of the break point occurring at $W_{0}=1 \mathrm{GHz}$ is confirmed from this plot, and intuitively a dual-slope regression fit is therefore appropriate. Note that a better approximation may be obtained with a higher-order polynomial, but we use a dual-slope model in the interest of simplicity and intuitiveness, and demonstrate that the simpler model also yields a good approximation. A slope-constant model is in fact adopted as a further simplification in this paper, given by

$$
F_{s \sigma}= \begin{cases}s\left(k_{1}-k_{2} \log _{10} W_{b}\right), & \text { if } W_{b}<W_{0} \\ s k_{3}, & \text { otherwise }\end{cases}
$$

where $s=\{1,2,3\}$ denotes the number of standard deviations considered in $F_{s \sigma}$. The model parameters $k_{1}$ and $k_{2}$, corresponding to the linear region $W_{b}<W_{0}$, are obtained as the intercept and slope of the regression line defined by $\log _{10} W_{b}$ versus $F_{s \sigma}$, while $k_{3}$ characterizes the fade depth at $W>W_{0}$. Thus from (5), we obtain the model parameters $k_{1}$ and $k_{2}$ as

$$
k_{1}=\left.\frac{F_{s \sigma}}{s}\right|_{W_{b} \rightarrow 1} ; \quad k_{2}=\frac{s k_{1}-F_{s \sigma}}{s \log _{10} W_{b}},
$$

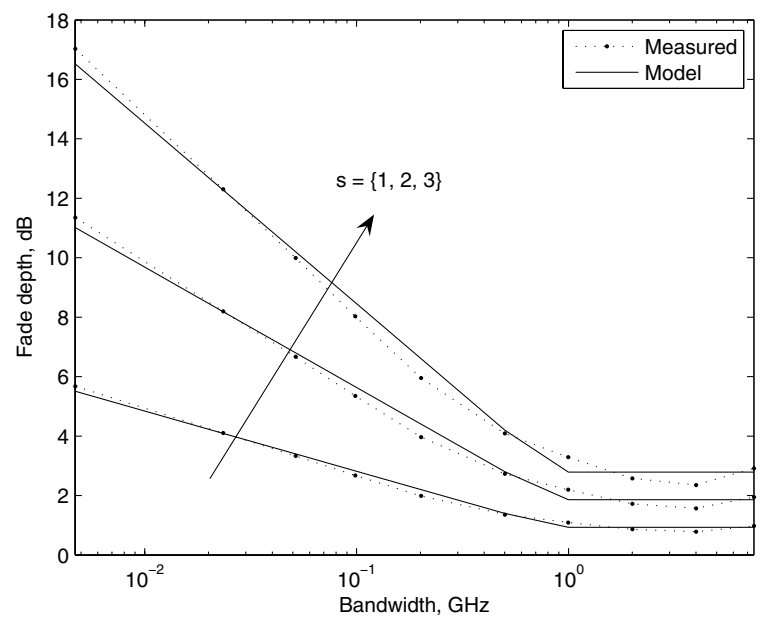

Fig. 6. A dual-slope model for the prediction of fade depth, $F_{s \sigma}$, for various values of $s$. The measured fade depth is also shown for comparison.

TABLE I

EMPIRICAL FADE DEPTH MODEL PARAMETERS

\begin{tabular}{ccccc}
\hline \hline \multirow{2}{*}{ Parameter } & \multicolumn{2}{c}{ Vertical Polarization } & \multicolumn{2}{c}{ Horizontal Polarization } \\
\cline { 2 - 5 } & LOS & NLOS & LOS & NLOS \\
\hline$k_{1}$ & 5.6 & 6.3 & 7.1 & 5.7 \\
$k_{2}$ & 1.7 & 2.0 & 2.2 & 1.8 \\
$k_{3}$ & 1.4 & 0.9 & 1.7 & 1.1 \\
\hline \hline
\end{tabular}

while the parameter $k_{3}$ is evaluated as

$$
k_{3}=\left.\frac{1}{n_{i}} \sum_{i=1}^{n_{i}} F_{s \sigma}\right|_{W_{b} \geq W_{0}} .
$$

The values of the three parameters, obtained empirically for a variety of propagation channel types, are listed in Table I. An inspection of Table I reveals that the intercept parameter, $k_{1}$, is somewhat larger in NLOS VV and LOS HH channels compared to the LOS VV and NLOS HH channels. A larger value of the intercept implies a greater fade depth and consequent power loss at the receiver when the channel bandwidth is vanishingly small. Thus, from Table I, an indoor narrowband system, with $W_{b} \rightarrow 1 \mathrm{~Hz}$ and vertical polarization, will experience $F_{3 \sigma}=17$ and $19 \mathrm{~dB}$ when operated in LOS and NLOS conditions, respectively. As $W_{b}$ increases, the difference in the values of $F D_{\sigma}$ for various signal polarizations and propagation conditions is progressively reduced in accordance with (5). In a similar manner, the difference between the values of the slope parameter, $k_{2}$ is insignificant, as observed from Table I. The large-bandwidth fade depth parameter, $k_{3}$, varies by up to $0.8 \mathrm{~dB}$ for the various propagation conditions in Table I. Based on these observation, we can infer that the fade depth in the measured indoor environments is nearly independent of the signal polarization and LOS conditions, especially for the case of wideband and UWB channels.

Fig. 6 shows close agreement between the measured and predicted fade depth for the NLOS VV channel. The prediction 
error at $W_{0}$ is $0.5 \mathrm{~dB}$ for $F_{3 \sigma}$ and lower for smaller $s$. The asymptotic error at large $W_{b}$ between the measured and predicted $F_{3 \sigma}$ is $0.1 \mathrm{~dB}$. The prediction errors for the $\mathrm{HH}$ polarization and for the LOS channels are of the same order. This close agreement between the measurements and the proposed model illustrates the accuracy of our simple model relating the fade depth to channel bandwidth.

\section{CONCLUSiON}

The relationship between the channel bandwidth and fade depth has been established in this paper. According to the observations from our indoor measurements, the fade depth, expressed in $\mathrm{dB}$, scales down linearly with log-bandwidth up to $1 \mathrm{GHz}$, and is nearly constant thereafter. We thus find that the frequency diversity of the channel is saturated as the bandwidth exceeds $1 \mathrm{GHz}$. The empirical dual-slope model accurately describes the dependence of fade depth on bandwidth, with a maximum error of $0.5 \mathrm{~dB}$ for the threestandard-deviation fade depth evaluation. Our analysis has also shown that the link polarization or presence of line-of-sight does not affect the fade depth significantly, especially in the wideband and UWB channels. The precise values of the model parameters may vary slightly from those reported in this paper when the measurements are conducted in other environments. The overall trends, however, and indeed the dual-slope model itself, are expected to be valid for a variety of configurations.

\section{ACKNOWLEDGMENT}

This work was supported in part by the UK Engineering and Physical Sciences Research Council via Grant GR/T21769/01.

\section{REFERENCES}

[1] T. S. Rappaport, Wireless Communications: Principles and Practice, 2nd ed. Prentice Hall, 2001.

[2] D. Tse and P. Viswanath, Fundamentals of Wireless Communication. Cambridge, UK: Cambridge University Press, 2005.

[3] B. Allen, M. Dohler, E. E. Okon, W. Q. Malik, A. K. Brown, and D. J. Edwards, Eds., Ultra-Wideband Antennas and Propagation for Communications, Radar and Imaging. London, UK: Wiley, 2006.

[4] U. G. Schuster, H. Bölcskei, and G. Durisi, "Ultra-wideband channel modeling on the basis of information-theoretic criteria," in Proc. IEEE Int. Symp. Inf. Theory, Adelaide, Australia, Sept. 2005.

[5] A. M. Hayar, R. Knopp, and R. Saadane, "Subspace analysis of indoor UWB channels," EURASIP J. Appl. Sig. Proc., no. 3, 2005.

[6] J. Romme and B. Kull, "On the relation between bandwidth and robustness of indoor UWB communication," in Proc. IEEE Conf. Ultra Wideband Sys. Tech. (UWBST), Reston, VA, USA, Nov. 2003.

[7] R. L. Olsen and T. Tjelta, "Worldwide techniques for predicting the multipath fading distribution on terrestrial LOS links: background and results of tests," IEEE Trans. Antennas Propagat., vol. 47, no. 1, Jan. 1999.

[8] P. Wilson, P. Papazian, M. Cotton, and Y. Lo, "A comparison of 1920$\mathrm{MHz}$ mobile channel diversity gain using horizontal and vertical arrays," IEEE Trans. Commun., vol. 49, no. 12, Dec. 2001.

[9] A. M. Street, L. Lukama, and D. J. Edwards, "Use of VNAs for wideband propagation measurements," IEE Proc.-Commun., vol. 148, no. 6, Dec. 2001

[10] W. Q. Malik, D. J. Edwards, and C. J. Stevens, "Angular-spectral antenna effects in ultra-wideband communications links," IEE Proc.-Commun., vol. 153, no. 1, Feb. 2006.

[11] R. W. P. King, Theory of Linear Antennas. Cambridge, MA, USA: Harvard Press, 1956.

[12] P. Pagani and P. Pajusco, "On the fading properties of a UWB link in a dynamic environment," in Proc. Eur. Electromagnetics Symp., Magdeburg, Germany, July 2004.

[13] Z. Tarique, W. Q. Malik, and D. J. Edwards, "Bandwidth requirements for accurate detection of direct path in multipath environment," Electon. Lett., vol. 42, no. 2, Jan. 2006. 